
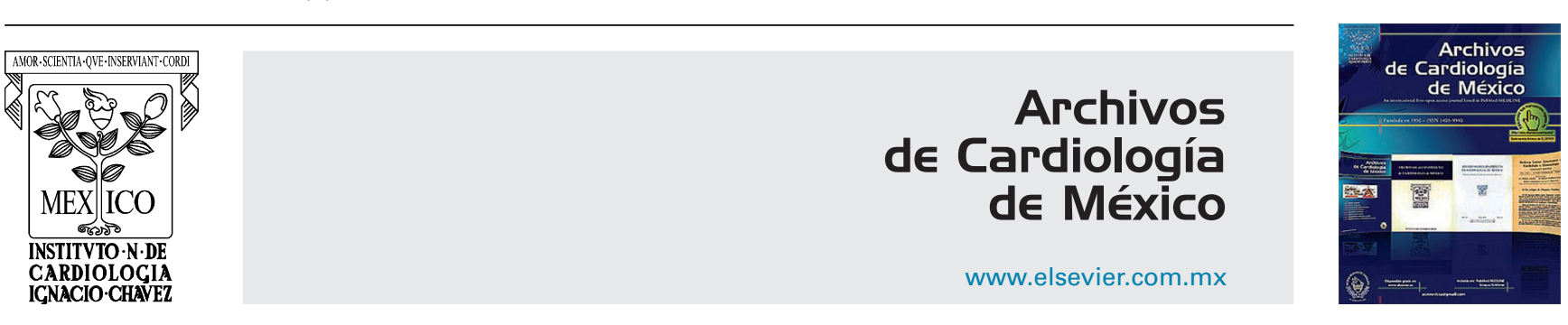

INVESTIGACIÓN CLÍNICA

\title{
Características electrocardiográficas de vías fascículo-ventriculares en pediatría: estudio comparativo con las vías accesorias anteroseptales derechas
}

\author{
Julián Andrés Olmedo ${ }^{\mathrm{a}, *}$, Mauricio Sebastián Abello ${ }^{\mathrm{a}}$, Bryan Cannon ${ }^{\mathrm{b}}$, \\ David Doiny $^{a}$, Javier Bonacina ${ }^{a}$ y José Manuel Moltedo ${ }^{a}$ \\ a Servicio de Electrofisiología, Departamento de Cardiología, Instituto FLENI, Buenos Aires, Argentina \\ b Departamento de enfermedades cardiovasculares pediátricas y adolescentes, Mayo Clinic, , Minneapolis, Minnesota, Estados \\ Unidos
}

Recibido el 11 de enero de 2017; aceptado el 29 de mayo de 2017

\author{
PALABRAS CLAVE \\ Vías accesorias fascí- \\ culoventriculares; \\ Síndrome de WPW; \\ Pediatría; \\ Argentina
}

\begin{abstract}
Resumen
Objetivos: Las vías accesorias (VAc) fascículo-ventriculares (FV) tienen una localización anatómica similar a las VAc anteroseptales derechas (ASD) y comparten características electrocardiográficas. El objetivo es comparar características electrocardiográficas de las VAC FV con las de las ASD en pediatría.

Métodos: Se incluyeron pacientes con preexcitación manifiesta sometidos a estudio electrofisiológico. Las VAc FV se definieron por un intervalo $\mathrm{HV} \leq 32 \mathrm{~ms}$ y un alargamiento del $\mathrm{AH}$ sin modificación del HV, del grado o patrón de preexcitación ventricular durante la estimulación auricular. Tres observadores independientes y ciegos analizaron los ECG en cada grupo.

Resultados: De 288 pacientes, 15 (5.2\%) presentaban VAC FV y 14 VAC ASD (4.9\%). El intervalo PR fue más largo en las VAc FV que en las ASD $(113 \pm 21$ vs. $86 \pm 13$ ms respectivamente; $p=<0.001)$ y la duración del QRS fue menor ( $95 \pm 12$ vs. $137 \pm 24$ ms respectivamente; $p=<0.001)$. El ECG de las VAc FV presentó una deflexión rápida de baja amplitud previa al inicio del QRS en 13 de 15 pacientes (87\%) y en 2 con VAc AV ASD $(14 \%) ;(p=0.003)$.

Conclusiones: El intervalo PR fue más largo y el complejo QRS más angosto en la VAC FV respecto de las ASD. La presencia de una deflexión rápida de baja amplitud previa al inicio del QRS permitiría diferenciarlas de las aurículo-ventriculares ASD de manera no invasiva.

(C) 2017 Instituto Nacional de Cardiología Ignacio Chávez. Publicado por Masson Doyma México S.A. Este es un artículo Open Access bajo la licencia CC BY-NC-ND (http://creativecommons. org/licenses/by-nc-nd/4.0/).
\end{abstract}

\footnotetext{
* Autor para correspondencia: Montañeses 2325, Ciudad de Buenos Aires. Argentina. CP: 1428. Teléfono: +54-011-1535022041; fax: +57773209 .

Correo electrónico: julianolm5@yahoo.com.ar (J.A. Olmedo). 


\section{KEYWORDS}

Fasciculo-ventricular accessory pathways; WPW syndrome; Children; Argentina
Electrocardiographic characteristics of fasciculo-ventricular accessory pathways in children: A comparative study with right anteroseptal accessory pathways

\begin{abstract}
Objectives: Fasciculo-ventricular (FV) accessory pathways (AP's) and right anteroseptal (RAS) AP's share similar anatomic locations and electrocardiographic characteristics. The objective of this article is to compare these features in children.

Methods: All patients with manifest pre-excitation who underwent an electrophysiological study were included. Fasciculo-ventricular AP's were defined by the presence of an HV inter$\mathrm{val} \leq 32 \mathrm{~ms}$ and a prolongation of the $\mathrm{AH}$ without changes in the HV interval, or the level of pre-excitation during atrial pacing. Three independent and blind observers analysed the ECG's in both groups.

Results: Out of 288 patients, 15 (5.2\%) had FV AP's and 14 (4.9\%) right AS AP's. The PR interval was longer in FV AP's than in RAS $(113 \pm 21$ vs $86 \pm 13$ ms respectively; $P<.001)$ and the QRS was narrower ( $95 \pm 12$ vs $137 \pm 24$ ms respectively; $P<.001$ ). The ECG in patients with FV AP's showed a rapid low amplitude deflection at the begining of the QRS in 13 out of 15 patients $(87 \%)$ and in $2(14 \%)$ the RAS AP group $(P=.003)$.

Conclusions: The PR interval was longer and the QRS complex was narrower in patients with FV AP's. The presence of a rapid low amplitude deflection at the beginning of the QRS complex would allow to differentiate them from RAS AP's non-invasively.

(C) 2017 Instituto Nacional de Cardiología Ignacio Chávez. Published by Masson Doyma México S.A. This is an open access article under the CC BY-NC-ND license (http://creativecommons. org/licenses/by-nc-nd/4.0/).
\end{abstract}

\section{Introducción}

Las vías accesorias (VAc) fascículo-ventriculares (FV) son una variante rara de preexcitación ventricular con una prevalencia reportada en la población pediátrica del 1.2-5.1\% ${ }^{1-5}$. Se manifiestan electrocardiográficamente por un intervalo PR normal o variable, la presencia de una onda delta y un complejo QRS relativamente estrecho. Desde el punto de vista electrofisiológico presentan un intervalo HV corto y fijo, sin evidenciarse cambios en el grado de preexcitación ventricular durante estimulación auricular (fisiología de Mahaim) ${ }^{1-8}$. Se considera que estas vías no forman parte activa de circuitos de taquicardias reentrantes aunque existen opiniones controvertidas al respecto 9 .

En la población adulta, se han observado semejanzas electrocardiográficas entre las VAc FV y las VAc anteroseptales derechas (ASD). Ambos grupos comparten polaridad positiva de los complejos QRS en derivaciones de la cara inferior y transición entre V1-V3, debido a una localización anatómica similar ${ }^{7,8}$. Sin embargo, no existen hasta la actualidad datos comparativos de ambos grupos en población pediátrica.

Los objetivos de este trabajo son determinar la prevalencia de las VAc FV en una población pediátrica con preexcitación ventricular, evaluar las características electrocardiográficas y compararlas con las de las VAc AV ASD en el mismo grupo etario.

\section{Métodos}

Se realizó un análisis retrospectivo de pacientes menores de 16 años consecutivos con preexcitación manifiesta (patrón de Wolff-Parkinson-White) en el ECG de superficie sometidos a estudio electrofisiológico y ablación por radiofrecuencia entre noviembre del 2004 y abril del 2013 de 2 centros, Instituto Fleni, Buenos Aires, Argentina, y Mayo Clinic Children's Center, Minnesota, EE. UU.

El estudio electrofisiológico fue realizado en todos los casos bajo anestesia general. Aquellos pacientes que recibían medicación antiarrítmica suspendieron la misma al menos 5 vidas medias previas de la intervención. Por acceso venoso femoral se posicionaron catéteres tetrapolares en la aurícula derecha alta, His, ápex del VD y en el seno coronario. Un catéter de ablación tetrapolar de $4 \mathrm{~mm}$ fue utilizado tanto para el mapeo de las VAc FV como de las VAc ASD.

Se realizaron mediciones del intervalo PR, del complejo $\mathrm{QRS}$, así como de los intervalos AH y HV en ritmo sinusal. Las mediciones del ECG de superficie se realizaron en la derivación DII, en forma digitalizada mediante el polígrafo de electrofisiología a velocidad de $200 \mathrm{~mm} / \mathrm{seg}$.

El registro del hisiograma debía presentar una deflexión auricular con una relación AV 1:4. Se realizaron mediciones de los intervalos $\mathrm{AH}$ y $\mathrm{HV}$ en ritmo sinusal con calipers a una velocidad de $100 \mathrm{~mm} / \mathrm{seg}$.

Se consideraron como VAc FV aquellas que presentaran los siguientes criterios electrofisiológicos:

1) Intervalo $\mathrm{HV} \leq 32 \mathrm{~ms}$ en ritmo sinusal ${ }^{10}$.

2) Alargamiento progresivo del intervalo $\mathrm{AH}$ sin modificación del intervalo $\mathrm{HV}$, ni del grado o patrón de preexcitación ventricular durante la estimulación auricular programada con trenes más un extraestímulo con acoplamientos progresivamente más cortos.

Los criterios diagnósticos considerados para los pacientes con VAc AV ASD fueron: intervalo $\mathrm{HV} \leq 32 \mathrm{~ms}$ en ritmo sinusal con aumento progresivo de la preexcitación ventricular ante 
la estimulación auricular; y durante el mapeo de la vía, una delta-V más precoz entre hora 1 y 2 del anillo tricuspídeo.

Se midieron los períodos refractarios efectivos del nodo AV (NAV) y VAc. En los pacientes que presentaban VAc AV o taquicardias reentrantes nodales asociadas a las VAc FV, las mediciones fueron realizadas luego de las ablaciones de estos sustratos. Para completar el diagnóstico, a los pacientes con VAc FV se les administró un bolo de $0.1-0.2 \mathrm{mg} / \mathrm{kg}$ de adenosina intravenosa durante estimulación auricular con el objetivo de conseguir bloqueo transitorio del NAV y descartar presencia de otras VAc.

Se realizó la comparación de las características clínicas, electrocardiográficas y electrofisiológicas entre los pacientes con VAc FV y AV ASD. Finalmente un grupo de observadores independientes y ciegos analizaron los hallazgos electrocardiográficos de cada grupo.

\section{Análisis estadístico}

Los valores numéricos fueron expresados en media \pm desviación estándar. Se utilizaron pruebas de Chi cuadrado para las variables categóricas y test de Student para las numéricas. Los valores de $p$ menores a 0.05 fueron considerados como significativos.

\section{Resultados}

\section{Características clínicas de los pacientes con vías fascículo-ventriculares}

De un total de 288 pacientes con VAc, 15 (5.2\%) presentaban vías $\mathrm{FV}$. Seis de ellos (40\%) eran varones y la edad media fue de $12 \pm 3$ años (rango: 6-16). Todos los pacientes con vías FV tenían corazón estructuralmente sano, con excepción de un paciente con miocardiopatía hipertrófica y otro con insuficiencia mitral congénita. De los 15 pacientes con VAc FV, 10 (66\%) presentaban palpitaciones previo al procedimiento, todos ellos debido a un segundo sustrato arrítmico como se detallará más adelante, mientras que el resto estaba asintomático y el diagnóstico de la preexcitación fue incidental.

\section{Hallazgos electrocardiográficos y electrofisiológicos en pacientes con vías fascículo-ventriculares}

El intervalo PR medio fue de $113 \pm 21 \mathrm{~ms}$ [88-161 ms] y la duración media del QRS de $95 \pm 12 \mathrm{~ms}$ [80-120 ms]. En todos los pacientes, la polaridad de la onda delta y del QRS fue positiva en DI y en cara inferior (DII, DIII y AVF). Nueve pacientes $(60 \%)$ presentaban un patrón $\mathrm{rS}$ y el resto $(40 \%)$ un patrón $\mathrm{S}$ en la derivación V1. La transición precordial del QRS fue en V3 en 9 pacientes (60\%) y en V2 en los 6 restantes (40\%).

Se observó entre el segmento PR y el inicio de la onda delta una deflexión rápida de baja amplitud en 13 de 15 (87\%) pacientes (fig. 1). En 10 de ellos dicha señal se observó en la derivación V1 (77\%) y en 4 (30\%) en derivaciones de la cara inferior ( 3 en DIII y uno en AVF). En un paciente se presentó simultáneamente en V1 y en DIII (fig. 2).

Desde el punto de vista electrofisiológico, el intervalo $\mathrm{AH}$ basal medio fue de $73 \pm 21 \mathrm{~ms}$ y el intervalo $\mathrm{HV}$ medio de $20 \pm 6 \mathrm{~ms}$. Mediante la estimulación desde el ápex del VD se evidenció conducción ventrículo-auricular (V-A) concéntrica y decremental por el NAV en 12 pacientes (80\%); 3 presentaban disociación $\mathrm{V}$-A. El período refractario efectivo anterógrado del NAV fue de $330 \pm 130 \mathrm{~ms}$. En ningún paciente se indujo taquicardia mediada por la vía FV.

Se indujeron taquicardia por reentrada nodal típica en 4 pacientes, mientras que en otros 4 se indujeron taquicardias
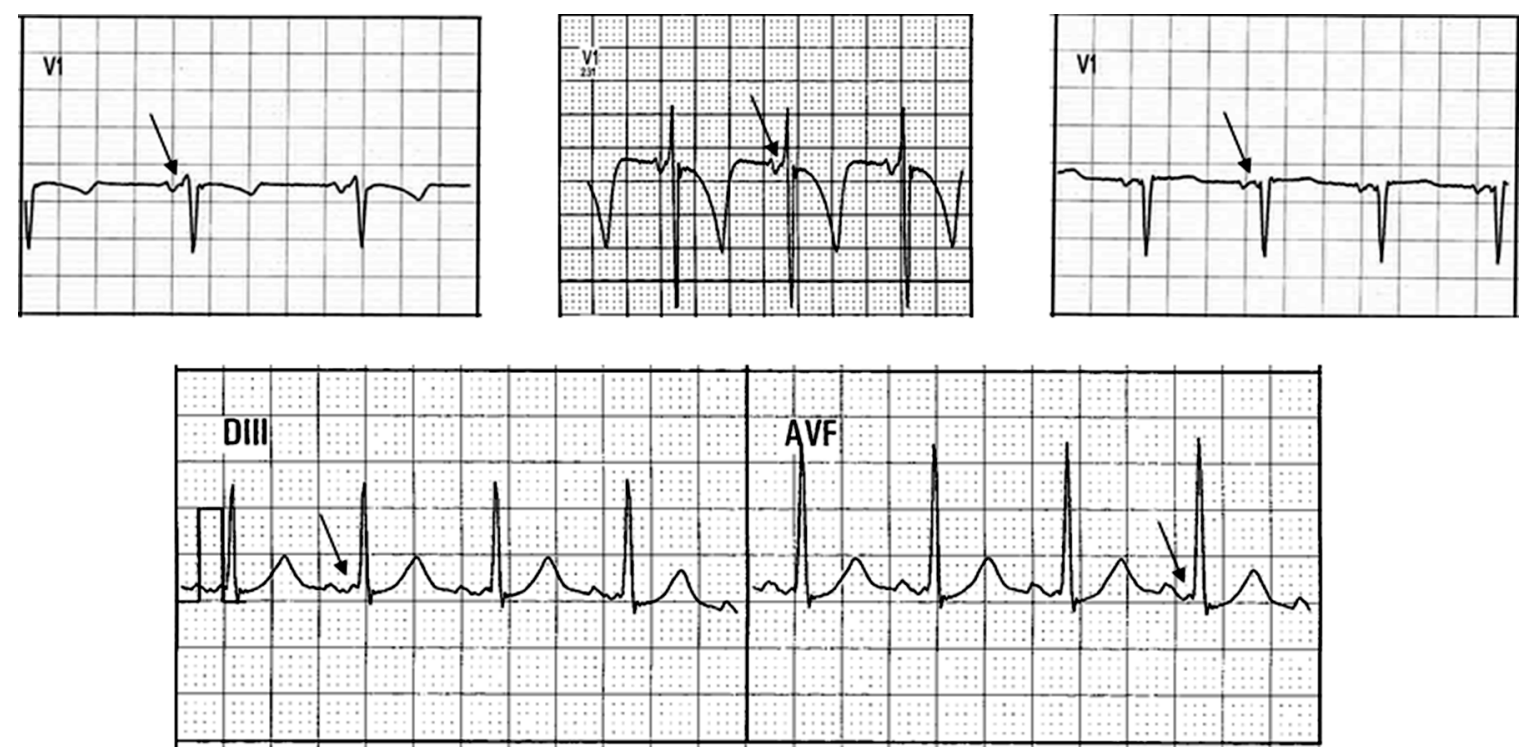

Figura 1 Se muestran 4 ejemplos de pacientes con VAC FV en los cuales la duración de los intervalos PR y complejos QRS son cercanos a los valores normales para la edad. Entre el segmento PR y el inicio de la onda delta se evidencia una deflexión rápida de baja amplitud (fecha), principalmente en la derivación V1 (hilera superior) y en derivaciones DIII y AVF (hilera inferior) a la que denominamos «potencial de vía».

VAC FV: vías accesorias fascículo-ventriculares. 

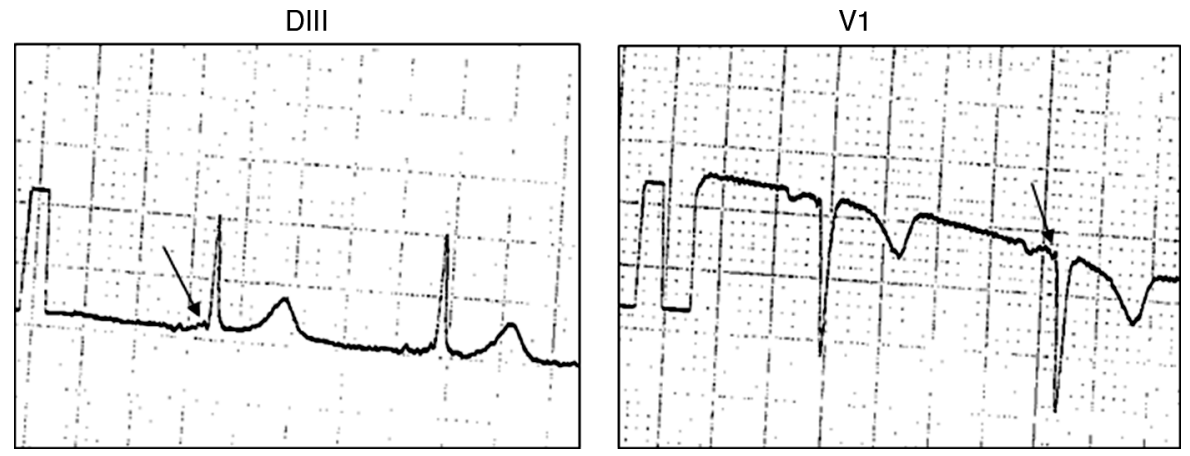

Figura 2 Presencia de un «potencial de vía» (flecha) simultáneamente en V1 y en DIII en un paciente con VAc FV. VAc FV: vía accesoria fascículo-ventricular.

por reentrada AV ortodrómicas por la presencia de VAC asociadas. En 2 pacientes coexistieron ambos mecanismos de taquicardia.

Seis pacientes (40\%) presentaron otras VAc AV de conducción manifiesta asociadas; 4 de localización izquierda (posterolateral, posteroseptal, anteroseptal y anterolate$\mathrm{ral})$, mientras que uno era derecha lateral. El paciente con miocardiopatía hipertrófica presentó VAc AV múltiples (2 derechas y 2 izquierdas).

Durante el mapeo de las VAc FV con el catéter de ablación, se evidenció una mayor precocidad delta-V en la región anteroseptal en 14 pacientes. En el restante, la mayor precocidad se obtuvo en los polos proximales del seno coronario, pudiéndose constatar una inserción posteroseptal izquierda de la VAc tras acceder al anillo mitral a través de una fosa oval permeable.

La respuesta a la infusión en bolo de adenosina fue bloqueo $\mathrm{AV}$ en todos los casos, excepto en un paciente en quien se evidenció una prolongación del intervalo PR a expensas del intervalo $\mathrm{AH}$, sin observarse cambios en la morfología del QRS. En los que tuvieron bloqueo del AV con la administración de adenosina se observó también la desaparición transitoria de la deflexión rápida previa al inicio del QRS antes mencionada.

Durante el seguimiento a mediano plazo, un paciente evidenció la pérdida de la preexcitación ventricular mediada por una VAC FV (fig. 3).

\section{Características clínicas, electrocardiográficas y electrofisiológicas de los pacientes con vías accesorias aurículo-ventriculares anteroseptales derechas}

De los 288 pacientes incluidos, 14 tenían VAc AV ASD (4.9\%). Diez $(71 \%)$ eran varones con una media de edad de $12 \pm 2$ años [8-15 años]. Todos tenían corazón estructuralmente normal. Ocho de los 14 pacientes (57\%) presentaban palpitaciones previas al procedimiento.

Los intervalos PR y QRS fueron de $86 \pm 13 \mathrm{~ms}$ [69-106 ms] y $137 \pm 24 \mathrm{~ms}$ [98-185 ms] respectivamente. Todos presentaron onda $\mathrm{R}$ y delta positiva en DI y en cara inferior (DII, DIII y AVF), la transición precordial del QRS fue en V3 en 10 pacientes $(71 \%)$, y en V2 en los 4 restantes $(29 \%)$. Se observó en DIII una señal rápida de baja amplitud inmediatamente antes del inicio del QRS en 2 de los 14 pacientes
(14\%), que desapareció luego de la ablación exitosa de la VAc. Un paciente presentó una segunda vía de conducción manifiesta de pared libre derecha y las mediciones del ECG de superficie se realizaron una vez eliminada esta vía.

Los intervalos $\mathrm{AH}$ y $\mathrm{HV}$ fueron de $65 \pm 18 \mathrm{~ms}$ y $-1.5 \pm 14 \mathrm{~ms}$ respectivamente. El período refractario efectivo anterógrado medio de las VAc AV ASD fue de $370 \pm 110 \mathrm{~ms}$ y del NAV de $330 \pm 100 \mathrm{~ms}$. Se indujeron taquicardias por reentrada AV ortodrómicas en 2 pacientes durante el protocolo de estimulación.

\section{Comparación de los pacientes con vías fascículo-ventriculares y vías aurículo-ventriculares anteroseptales derechas (tabla 1)}

No hubo diferencias significativas entre ambos grupos respecto a la edad, género y manifestación clínica. El intervalo PR fue más largo en las VAc FV que en las AV ASD $(113 \pm 21$ vs. $86 \pm 13$ ms respectivamente; $p<0.001)$, mientras que la duración del complejo QRS fue menor ( $95 \pm 12$ vs. $137 \pm 24$ ms respectivamente; $p<0.001$ ), similar a lo reportado en adultos ${ }^{7,8}$. No existieron diferencias significativas en la polaridad de la onda delta, ni en la transición del intervalo QRS en las derivaciones precordiales (fig. 4).

Los pacientes con VAc FV presentaron una deflexión rápida de baja amplitud previa al inicio del complejo QRS en 13 de 15 pacientes (87\%) y en 2 en el grupo de las VAc AV ASD $(14 \%)(p=0.003)$.

Tabla 1 Tabla comparativa que muestra las principales diferencias electrocardiográficas y electrofisiológicas entre ambos grupos: vías FV y WPW ASD

\begin{tabular}{llll}
\hline & Vía FV & WPW ASD & $\mathrm{p}$ \\
\hline Edad & $11.6 \pm 3.2$ & $11.7 \pm 2.6$ & $\mathrm{n} / \mathrm{s}$ \\
PR (ms) & $113 \pm 21$ & $86 \pm 13$ & 0.0001 \\
QRS (ms) & $95 \pm 12$ & $137 \pm 24$ & $<0.0001$ \\
Muesca pre-QRS & SI $13 / 15(87 \%)$ & SI $2 / 14(14 \%)$ & 0.003 \\
AH basal (ms) & $73 \pm 21$ & $65 \pm 19$ & $\mathrm{n} / \mathrm{s}$ \\
HV basal (ms) & $20 \pm 6$ & $-1.50 \pm 14$ & $<0.001$ \\
\hline
\end{tabular}

FV: fascículo-ventricular; WPW ASD: Wolff-Parkinson-White con vía accesoria anteroseptal derecha. 

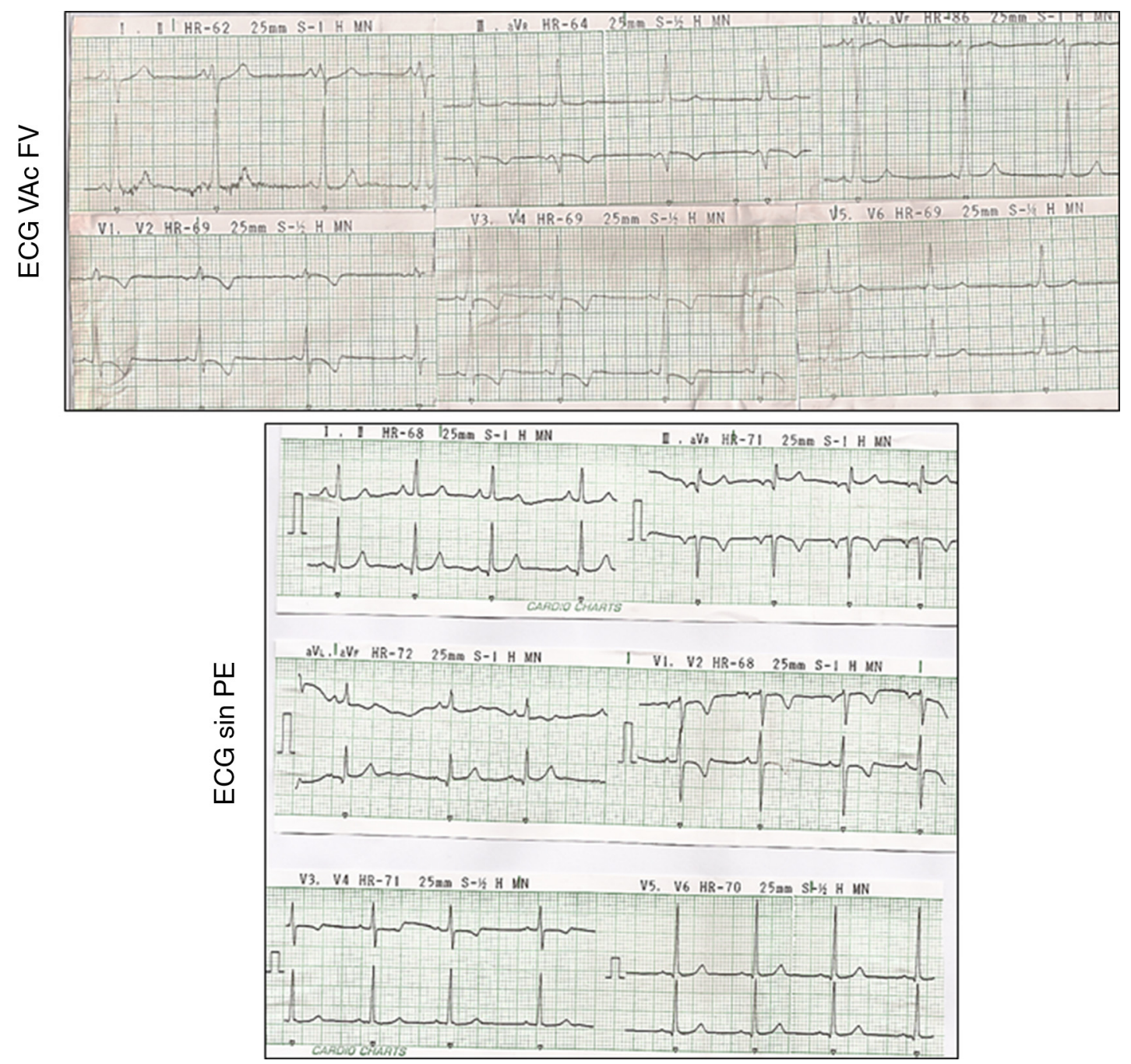

Figura 3 ECG superior que muestra PE perteneciente al paciente con VAc FV de inserción izquierda (ver texto); y la desaparición de misma durante el seguimiento del mismo paciente (ECG inferior).

PE: preexcitación ventricular; VAc FV: vía accesoria fascículo-ventricular.

Los pacientes con VAc FV presentaron intervalos HV basal más largos que los que tenían VAc AV ASD (20 \pm 6 vs. $-1.5 \pm 14$ ms respectivamente; $p<0.001)$.

\section{Discusión}

La presente serie de pacientes pediátricos con vías FV diagnosticadas mediante estudio electrofisiológico es la más grande reportada en la literatura hasta la actualidad. La prevalencia de vías $\mathrm{FV}$ en nuestra población de pacientes con preexcitación ventricular es del 5.2\%, similar a la reportada en la bibliografía ${ }^{1-3}$. Probablemente, la prevalencia de las vías FV esté subestimada debido a su bajo nivel de sospecha determinada por su menor grado de preexcitación, intervalos PR y duración del QRS cercanos a los valores normales y al curso asintomático de esta enfermedad.

La asociación de vías FV con otras VAc en nuestra serie de pacientes fue similar a la reportada anteriormente (un $40 \%$ a otras VAc y un $10 \%$ a vías múltiples) $)^{2,3,11-13}$.

La identificación electrocardiográfica de las vías FV y su diferenciación con las VAc AV ASD es dificultosa, requiriendo en la mayoría de los casos un diagnóstico invasivo ${ }^{1,2}$. Hasta el momento solo existen 2 reportes comparativos entre las VAc FV y las AV ASD en adultos ${ }^{7,8}$, siendo este el primero que las diferencia en la población pediátrica.

Sternick et al. ${ }^{8}$ compararon las vías FV, VAc AV ASD y medioseptales y observaron que en las VAc FV la transición en las derivaciones precordiales era principalmente en V2, a diferencia de la presente serie en la cual se evidencia mayoritariamente en V3. En el mismo trabajo demostraron que solo un $25 \%$ de las vías medioseptales presentaron onda delta positiva en la cara inferior, a diferencia de las vías FV y ADS en las que fue positiva en el $100 \%$ de los casos. Esta diferencia electrocardiográfica motivó a no incluir pacientes con VAc medioseptales en nuestro trabajo ya que se las podía diferenciar de manera no invasiva.

En los ECG de superficie identificamos previo al inicio de la onda delta una deflexión rápida de baja amplitud en aquellos pacientes con VAc FV que denominamos «potencial de vía». Este hallazgo se hace más evidente en las derivaciones precordiales septales debido, hipotéticamente, a la proximidad cardíaca a estas derivaciones con el sitio donde se inserta la vía. 

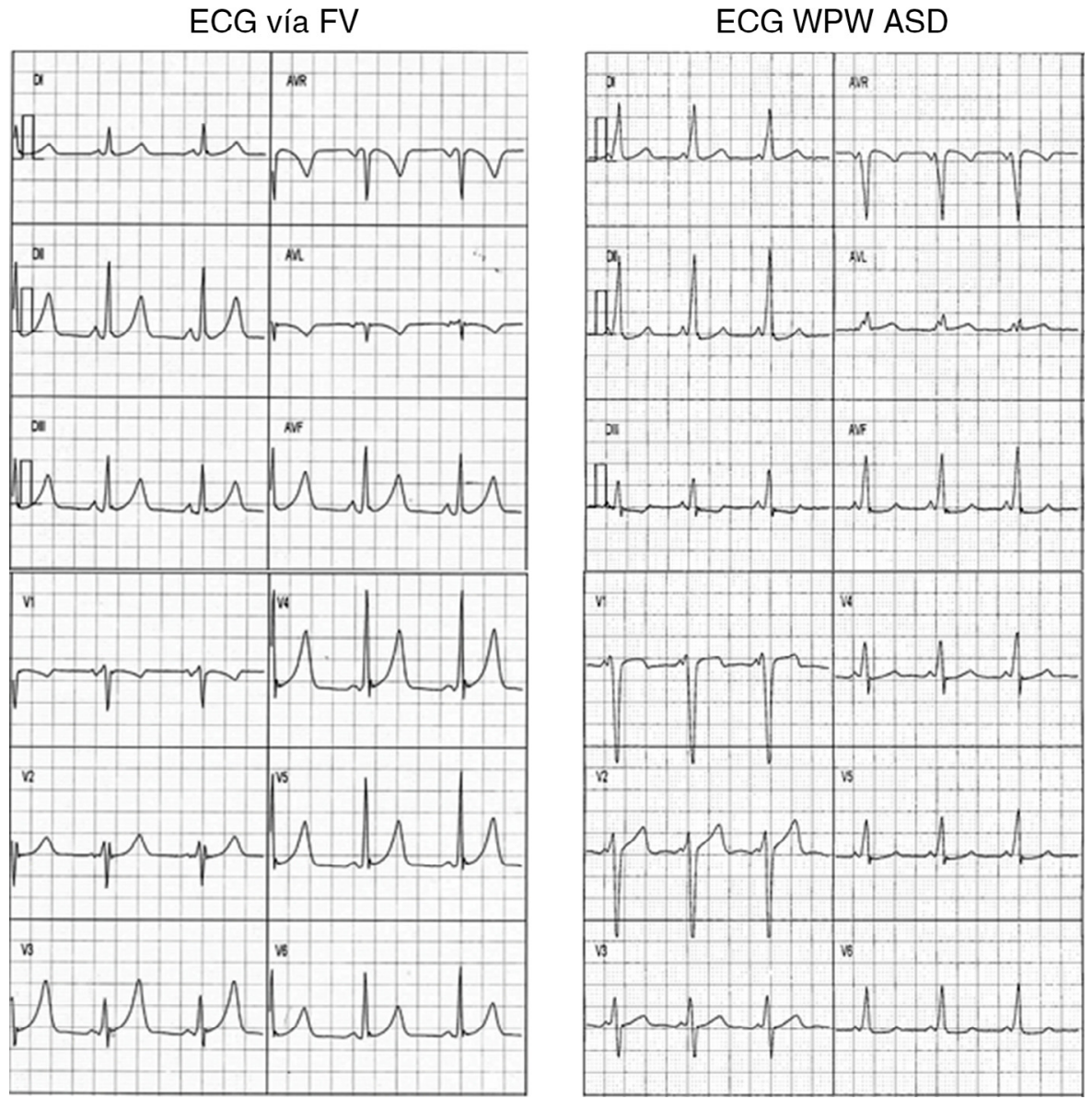

Figura 4 Se muestran 2 ejemplos de ECG de 12 derivaciones de un paciente con vía FV (izquierda) y otro con WPW ASD (derecha) observándose un intervalo PR y un QRS cercano a los límites normales para la edad en el ECG con vía FV. Por otro lado presentan similitudes tanto en la morfología del complejo QRS en cara inferior y en la derivación V1, su transición en la cara anterior, como en la polaridad de la onda delta en todas las derivaciones.

ASD: anteroseptal derecha; FV: fascículo-ventricular; WPW: Wolff-Parkinson-White.

El hecho de que se observe esta deflexión con más frecuencia en las VAc FV comparado con las ASD podría ser atribuido al «silencio eléctrico» otorgado por el retardo fisiológico del NAV (intervalo PR normal), a diferencia de un posible enmascaramiento dado por un intervalo PR más corto y la onda delta en las VAc AV ASD.

Este último hallazgo, junto a la presencia de un intervalo PR dentro de límites normales y un complejo QRS relativamente angosto, podría contribuir a la identificación más sencilla de estas vías, permitiendo adoptar una conducta conservadora en pacientes asintomáticos con mínima preexcitación.

Un paciente del grupo VAc FV evidenció la desaparición de la preexcitación ventricular durante el seguimiento, este hallazgo asociado a la baja prevalencia de estas vías en la población adulta ${ }^{2,7,8}$ puede indicar una involución anatómica y/o eléctrica de las vías FV con el tiempo.

\section{Conclusión}

Las vías FV tienen una prevalencia del 5.2\% en nuestra serie de pacientes pediátricos con preexcitación ventricular. La presencia de un intervalo PR y duración del complejo QRS cercanos a valores normales para la edad, sumado a una deflexión rápida de baja amplitud previo al inicio del QRS, podría permitir la identificación de estas vías y diferenciarlas de las aurículo-ventriculares ASD de manera no invasiva, permitiendo adoptar una conducta más conservadora en los pacientes asintomáticos.

\section{Responsabilidades éticas}

Protección de personas y animales. Los autores declaran que para esta investigación no se han realizado experimentos en seres humanos ni en animales.

Confidencialidad de los datos. Los autores declaran que han seguido los protocolos de su centro de trabajo sobre la publicación de datos de pacientes.

Derecho a la privacidad y consentimiento informado. Los autores declaran que en este artículo no aparecen datos de pacientes. 


\section{Financiamiento}

\section{Ninguno.}

\section{Conflicto de intereses}

Los autores declaran no tener ningún conflicto de intereses.

\section{Bibliografía}

1. Sallee D, van Hare GF. Preexcitation secondary to fasciculoventricular pathways in children: A report of three cases. J. Cardiovasc Electrophysiol. 1999;10:36-42.

2. Sternick EB, Gerken LM, Vrandecic M, et al. Fasciculoventricular pathways: Clinical and electrophysiologic characteristics of a variant of preexcitation. J. Cardiovasc Electrophysiol. 2003;14:1057-63.

3. Ratnasamy C, Khan D, Wolff GS, et al. Clinical and electrophysiological characteristics of fasciculoventricular fibers in children. Int J Cardiol. 2008;123:257-62.

4. Sternick EB, Oliva A, Gerken ML, et al. Clinical, electrocardiographic, and electrophysiologic characteristics of patients with a fasciculoventricular pathway: The role of PRKAG2 mutation. Heart Rhythm. 2011;8:58-64.

5. Govindan M, Ward D, Behr E. A rare conection: Fasciculoventricular pathway in PRKAG2 disease. J Cardiovasc Electrophysiol. 2010;21:329-32.
6. Josephson ME. Preexcitation syndromes. En: Josephson ME, editor. Clinical cardiac electrophysiology: Techinques and interpretations. 4th ed. Philadelphia: Lippicott Williams \& Wilkin; 2008. p. 440-2

7. Ho S, Choi YS, Choi EK, et al. Electrocardiographic characteristics of fasciculoventricular pathways. PACE. 2005;28: 25-8.

8. Sternick EB, Rodriguez LM, Gerken LM, et al. Electrocardiogram in patient whit fasciculoventricular pathway: A comparative study whit anteroseptal and midseptal accessory pathway. Heart Rhythm. 2005;2:1-6.

9. Cao K, Chen M, Zou J, et al. Narrow QRS tachycardia with ventriculoatrial dissociation mediated by a left fasciculoventricular fiber. J Interv Card Electrophysiol. 2005;13:151-7.

10. Gillette PC, Reitman MJ, Gutgesell HP, et al. Intracardiac electrography in children and young adults. Am Heart J. 1975;89:36-44

11. Colavita PG, Packer DL, Presselly JC, et al. Frequency, diagnosis and clinical characteristics of patients with multiple accessory atrioventricular pathways. Am J Cardiol. 1987;59: 601-6.

12. Weng KP, Wolff GS, Young ML. Multiple accessory pathway in pediatric patients with Wolff-Parkinson-White syndrome. Am J Cardiol. 2003;91:1178-83.

13. Vashist S, Silva JN, van Hare GF, et al. Unusually high association of hypertrophic cardiomyopathy and complex heart defects in children with fasciculoventricular pathways. PACE. 2012;35:308-13. 